\title{
NECESSARY AND SUFFICIENT CONDITIONS FOR COPOSITIVE TENSORS
}

\author{
Yisheng Song ${ }^{1,2}$ and Liqun $\mathrm{Qi}^{1}$ \\ 1. Department of Applied Mathematics, The Hong Kong Polytechnic \\ University, Hung Hom, Kowloon, Hong Kong \\ 2. College of Mathematics and Information Science, Henan Normal \\ University, XinXiang HeNan, P.R. China, 453007.
}

\begin{abstract}
In this paper, it is proved that a symmetric tensor is (strictly) copositive if and only if each of its principal subtensors has no (non-positive) negative $\mathrm{H}^{++}$-eigenvalue. Necessary and sufficient conditions for (strict) copositivity of a symmetric tensor are also given in terms of $Z^{++}$-eigenvalues of the principal sub-tensors of that tensor. This presents a method for testing (strict) copositivity of a symmetric tensor by means of lower dimensional tensors. Also an equivalent definition of strictly copositive tensors is given on the entire space $\mathbb{R}^{n}$.
\end{abstract}

Key Words and Phrases: Copositive Tensors, Principal sub-tensor, $H^{++}$-eigenvalue, $Z^{++}$-eigenvalue.

2010 AMS Subject Classification: 15A18, 15A69, 90C20, 90C30

\section{Introduction}

An $m$-order $n$-dimensional tensor $\mathcal{A}$ consists of $n^{m}$ entries in the real field $\mathbb{R}:$

$$
\mathcal{A}=\left(a_{i_{1} \cdots i_{m}}\right), \quad a_{i_{1} \cdots i_{m}} \in \mathbb{R}, \quad 1 \leq i_{1}, i_{2}, \cdots, i_{m} \leq n .
$$

Email: songyisheng1@gmail.com (Song); Liqun.Qi@polyu.edu.hk (Qi).

This work was supported by the Hong Kong Research Grant Council (Grant No. PolyU $501808,501909,502510,502111)$ and the first author was supported partly by the National Natural Science Foundation of P.R. China (Grant No. 11071279, 11171094, 11271112) and by the Research Projects of Science and Technology Department of Henan Province(Grant No. 122300410414 ). 
For $x=\left(x_{1}, x_{2}, \cdots, x_{n}\right)^{T} \in \mathbb{R}^{n}$ (or $\mathbb{C}^{n}$ ), $\mathcal{A} x^{m-1}$ is a vector in $\mathbb{R}^{n}$ (or $\mathbb{C}^{n}$ ) with its ith component defined by

$$
\left(\mathcal{A} x^{m-1}\right)_{i}=\sum_{i_{2}, \cdots, i_{m}=1}^{n} a_{i i_{2} \cdots i_{m}} x_{i_{2}} \cdots x_{i_{m}} .
$$

Then $x^{T}\left(\mathcal{A} x^{m-1}\right)$ is a homogeneous polynomial, denoted as $\mathcal{A} x^{m}$, i.e.,

$$
\mathcal{A} x^{m}=x^{T}\left(\mathcal{A} x^{m-1}\right)=\sum_{i_{1}, i_{2}, \cdots, i_{m}=1}^{n} a_{i_{1} i_{2} \cdots i_{m}} x_{i_{1}} x_{i_{2}} \cdots x_{i_{m}},
$$

where $x^{T}$ is the transposition of $x$. An $m$-order $n$-dimensional tensor $\mathcal{A}$ is called nonnegative (positive) if $a_{i_{1} i_{2} \cdots i_{m}} \geq 0\left(a_{i_{1} i_{2} \cdots i_{m}}>0\right)$ for all $i_{1}, i_{2}, \cdots, i_{m}$. An $m$-order $n$-dimensional tensor $\mathcal{A}$ is said to be symmetric if its entries $a_{i_{1} \cdots i_{m}}$ are invariant for any permutation of the indices. It is obvious that each $m$-order $n$-dimensional symmetric tensor $\mathcal{A}$ defines a homogeneous polynomial $\mathcal{A} x^{m}$ of degree $m$ with $n$ variables and vice versa.

As a natural extension of the counterparts for symmetric matrices, the concepts of eigenvalues and eigenvectors were introduced by Qi [18] for higher order symmetric tensors, and the existence of the eigenvalues and eigenvectors and their practical applications in determining positive definiteness of an even degree multivariate form were also studied by Qi [18]. Lim [12] independently introduced this notion and proved the existence of the maximum and minimum eigenvalues using a variational approach. Qi $[18,19]$ extended some nice properties of matrices to higher order tensors. Qi $[19,20]$ defined $E$-eigenvalues and the $E$-characteristic polynomial of a tensor, and proved that an E-eigenvalue of a tensor is a root of the $E$-characteristic polynomial. Subsequently, these topics attract attention of many mathematicians from different disciplines. For diverse studies and applications on these topics, see Chang [4], Chang, Pearson and Zhang [5], Chang, Pearson and Zhang [6], Hu, Huang and Qi [8], Hu and Qi [7], Ni, Qi, Wang and Wang [15], Ng, Qi and Zhou [16], Song and Qi [23, 24], Yang and Yang [26, 27], Zhang [28], Zhang and Qi [29], Zhang, Qi and Xu [30] and references cited therein.

For a vector $x \in \mathbb{R}^{n}, x \geq 0(x>0)$ means that $x_{i} \geq 0\left(x_{i}>0\right)$, $i=1,2, \cdots, n$. A real symmetric matrix $A$ is said to be (i) copositive if $x \geq 0$ implies $x^{T} A x \geq 0$; (ii) strictly copositive if $x \geq 0$ and $x \neq 0$ implies $x^{T} A x>0$. This concept is one of the most important concept in applied mathematics and graph theory, which was introduced by Motzkin [14] in 1952. In the literature, there are extensive discussions on such matrices. For example, Haynsworth and Hoffman [9] showed the Perron-Frobenius property of a copositive matrix; Martin [13] studied the properties of copositlve matrices by means of definiteness of quadratic forms subject to homogeneous linear inequality constraints; Väliäho [25] developed some finite criteria for (strictly) copositive matrices by searching its principal submatrices; Ping 
and $\mathrm{Yu}$ [17] obtained necessary and sufficient conditions for copositive matrices of order four; Kaplan [11] presented necessary and sufficient conditions for a symmetric matrix to be (strictly) copositive by using eigenvalues and eigenvectors of the principal submatrices of that matrix.

Theorem 1.1 (Kaplan [11]). Let $A$ be a symmetric matrix. Then $A$ is (strictly) copositive if and only if every principal submatrix $B$ of $A$ has no eigenvector $v>0$ with associated eigenvalue $(\lambda \leq 0) \lambda<0$.

One of the most important motivation for studying copositive matrices is that a large class of mixed-binary quadratic programs can be formulated as copositive programs [1] where a linear function is minimized over a linearly constrained subset of the cone of completely positive matrices, which is the dual cone of the copositive matrix cone. More recently, this equivalence has been extended to general nonconvex quadratically constrained quadratic program whose feasible region is nonempty and bounded [2]. Also there is a nice survey on copositive matrices and their applications [3]. It is interesting to see to what extent these results can be extend to the tensor. Recently, Qi [22] extended the concept of copositive matrices to tensors and found its many nice properties as copositive matrices. Suppose that a tensor $\mathcal{A}$ is a real symmetric tensor of order $m$ and dimension $n$. $\mathcal{A}$ is said to be

(i) copositive if $\mathcal{A} x^{m} \geq 0$ for all $x \in \mathbb{R}_{+}^{n}$;

(ii) strictly copositive if $\mathcal{A} x^{m}>0$ for all $x \in \mathbb{R}_{+}^{n} \backslash\{0\}$.

A matrix is a 2-order tensor, so it is very interesting to try and establish similar results for tensors as a parallel theory for matrices. The concept of principal sub-tensors of a symmetric tensor was introduced by Qi [18] for studying positive semidefiniteness of that tensor when the order of that tensor is even. Now we study the (strict) copositivity of a symmetric tensor $\mathcal{A}$ with the aid of the principal sub-tensors of $\mathcal{A}$.

In this paper, we will give an equivalent definition of (strict) copositivity of a symmetric tensor on the entire space $\mathbb{R}^{n}$. It is showed that a symmetric tensor $\mathcal{A}$ is (strictly) copositive if and only if every principal sub-tensor of $\mathcal{A}$ has no (non-positive) negative $H^{++}$-eigenvalues, i.e., every principal subtensor of $\mathcal{A}$ has no eigenvector $v>0$ with associated $H$-eigenvalue $(\lambda \leq 0)$ $\lambda<0$. The same conclusions still hold for using the $Z^{++}$-eigenvalue instead of $\mathrm{H}^{++}$-eigenvalue. Applying these results, we can test the copositivity of a symmetric tensor by means of lower dimensional tensors.

\section{Preliminaries and Basic facts}

In the sequel, we shall denote the transposition of a vector $x$ by $x^{T}$. Throughout this paper, let $\mathbb{R}_{+}^{n}=\left\{x \in \mathbb{R}^{n} ; x \geq 0\right\}, \mathbb{R}_{-}^{n}=\left\{x \in \mathbb{R}^{n} ; x \leq 0\right\}$, and $\mathbb{R}_{++}^{n}=\left\{x \in \mathbb{R}^{n} ; x>0\right\}$, and $e=(1,1, \cdots, 1)^{T}$. Denote by $e^{(i)}$ the

ith unit vector in $\mathbb{R}^{n}$, i.e., $e_{j}^{(i)}=1$ if $i=j$ and $e_{j}^{(i)}=0$ if $i \neq j$, for 
$i, j \in\{1,2, \cdots, n\}$.

Let $\mathcal{A}$ be an $m$-order $n$-dimensional tensor. A number $\lambda \in \mathbb{C}$ is called an eigenvalue of $\mathcal{A}$, if it and a nonzero vector $x \in \mathbb{C}^{n} \backslash\{0\}$ are solutions of the following systems of equations:

$$
\mathcal{A} x^{m-1}=\lambda x^{[m-1]},
$$

where $x^{[m-1]}=\left(x_{1}^{m-1}, \cdots, x_{n}^{m-1}\right)^{T}$, and call $x$ an eigenvector of $\mathcal{A}$ associated with the eigenvalue $\lambda$. We call such an eigenvalue $H$-eigenvalue if it is real and has a real eigenvector $x$, and call such a real eigenvector $x$ an $H$-eigenvector.

These concepts were first introduced by Qi [18] for higher order symmetric tensors. Lim [12] independently introduced this notion. Qi [18, 19] extended some nice properties of symmetric matrices to higher order symmetric tensors. The Perron-Frobenius theorem of nonnegative matrices had been generalized to higher order nonnegative tensors under various conditions by Chang, Pearson and Zhang [5], Hu, Huang and Qi [8], Yang and Yang [26, 27], Zhang [28] and others.

For an $m$-order $n$-dimensional tensor $\mathcal{A}$, we say a number $\mu \in \mathbb{C}$ is an E-eigenvalue of $\mathcal{A}$ and a nonzero vector $x \in \mathbb{C}^{n} \backslash\{\theta\}$ is an E-eigenvector of $\mathcal{A}$ associated with the $E$-eigenvalue $\mu$, if they are solutions of the following systems of equations:

$$
\left\{\begin{array}{l}
\mathcal{A} x^{m-1}=\mu x \\
x^{T} x=1 .
\end{array}\right.
$$

If $x$ is real, then $\mu$ is also real. In this case, $\mu$ and $x$ are called a $Z$ eigenvalue of $\mathcal{A}$ and a $Z$-eigenvector of $\mathcal{A}$ associated with the Z-eigenvalue $\mu$, respectively.

These concepts about E-eigenvalue were first introduced by Qi [18, 19] for studying the properties of a higher order tensor. Qi [20] defined the $E$-characteristic polynomial of a tensor $\mathcal{A}$, and showed that if $\mathcal{A}$ is regular, then a complex number is an E-eigenvalue of $\mathcal{A}$ if and only if it is a root of the E-characteristic polynomial.

Recently, Qi [21] introduced and used the following concepts for studying the properties of hypergraphs. An $H$-eigenvalue $\lambda$ of $\mathcal{A}$ is said to be (i) an $H^{+}$-eigenvalue of $\mathcal{A}$, if its $H$-eigenvector $x \in \mathbb{R}_{+}^{n}$;(ii) an $H^{++}$-eigenvalue of $\mathcal{A}$, if its $H$-eigenvector $x \in \mathbb{R}_{++}^{n}$. Similarly, we introduce the concepts of $Z^{+}$-eigenvalue and $Z^{++}$-eigenvalue. An $Z$-eigenvalue $\mu$ of $\mathcal{A}$ is said to be (a) a $Z^{+}$-eigenvalue of $\mathcal{A}$, if its $Z$-eigenvector $x \in \mathbb{R}_{+}^{n}$; (b) a $Z^{++}$-eigenvalue of $\mathcal{A}$, if its $Z$-eigenvector $x \in \mathbb{R}_{++}^{n}$. 


\section{Strictly Copositive Tensors with respect to entire space $\mathbb{R}^{n}$}

Let $\|\cdot\|$ denote any norm on $\mathbb{R}^{n}$. For $x=\left(x_{1}, x_{2}, \cdots, x_{n}\right)^{T}$, let

$$
x^{+}=\left(x_{1}^{+}, x_{2}^{+}, \cdots, x_{n}^{+}\right)^{T} \text { and } x^{-}=\left(x_{1}^{-}, x_{2}^{-}, \cdots, x_{n}^{-}\right)^{T},
$$

here $x_{i}^{+}=\max \left\{x_{i}, 0\right\}$ and $x_{i}^{-}=\max \left\{-x_{i}, 0\right\}$ for $i=1,2, \ldots, n$. Clearly, $x^{+} \geq 0, x^{-} \geq 0,\left|x_{i}\right|=x_{i}^{+}+x_{i}^{-}$, and $x=x^{+}-x^{-}$. Now we give the equivalent definition of (strict) copositivity of a symmetric tensor in the sense of any norm on $\mathbb{R}^{n}$.

Proposition 3.1. Let $\mathcal{A}$ be a symmetric tensor of order $m$ and dimension n. Then we have

(i) $\mathcal{A}$ is copositive if and only if $\mathcal{A} x^{m} \geq 0$ for all $x \in \mathbb{R}_{+}^{n}$ with $\|x\|=1$;

(ii) $\mathcal{A}$ is strictly copositive if and only if $\mathcal{A} x^{m}>0$ for all $x \in \mathbb{R}_{+}^{n}$ with $\|x\|=1$

(iii) $\mathcal{A}$ is strictly copositive if and only if $\mathcal{A}$ is copositive and the fact that $\mathcal{A} x^{m}=0$ for $x \in \mathbb{R}_{+}^{n}$ implies $x=0$.

Proof. (i) When $\mathcal{A}$ is copositive, the conclusion is obvious. Conversely, take $x \in \mathbb{R}_{+}^{n}$. If $\|x\|=0$, then it follows that $x=0$, and hence $\mathcal{A} x^{m}=0$. If $\|x\|>0$, then let $y=\frac{x}{\|x\|}$. We have $\|y\|=1$ and $x=\|x\| y$, and so

$$
\mathcal{A} x^{m}=\mathcal{A}(\|x\| y)^{m}=\|x\|^{m} \mathcal{A} y^{m} \geq 0 .
$$

Therefore, $\mathcal{A} x^{m} \geq 0$ for all $x \in \mathbb{R}_{+}^{n}$, as required.

Similarly, (ii) is easily proved.

(iii) Let $\mathcal{A}$ be strictly copositive. Clearly, $\mathcal{A}$ is copositive. Suppose there exists $x_{0} \in \mathbb{R}_{+}^{n}$ and $x_{0} \neq 0$ such that $\mathcal{A} x_{0}^{m}=0$, which contradicts the strict copositivity of $\mathcal{A}$. Conversely, if $x \neq 0$ and $x \in \mathbb{R}_{+}^{n}$, then $\mathcal{A} x^{m} \neq 0$. Since $\mathcal{A}$ is copositive, $\mathcal{A} x^{m}>0$. The conclusion follows.

Next we present the necessary and sufficient conditions of strict copositivity of a symmetric tensor on entire space $\mathbb{R}^{n}$.

Theorem 3.2. Let $\mathcal{A}$ be a symmetric tensor of order $m$ and dimension $n$. Then $\mathcal{A}$ is strictly copositive if and only if there is a real number $\gamma \geq 0$ such that

$$
\mathcal{A} x^{m}+\gamma\left\|x^{-}\right\|^{m}>0, \text { for all } x \in \mathbb{R}^{n} \backslash\{0\} .
$$

Proof. Let $\mathcal{A}$ be strictly copositive. Suppose that there is no $\gamma \geq 0$ such that the inequality (3.1) holds, i.e., for any real number $\gamma \geq 0$, there exists an $x^{(\gamma)} \in \mathbb{R}^{n} \backslash\{0\}$ such that

$$
\mathcal{A}\left(x^{(\gamma)}\right)^{m}+\gamma\left\|\left(x^{(\gamma)}\right)^{-}\right\|^{m} \leq 0 .
$$

In particular, for any positive integer $k$ (taking $\gamma=k$ ), there exists $x^{(k)} \in$ $\mathbb{R}^{n} \backslash\{0\}$ such that

$$
\mathcal{A}\left(x^{(k)}\right)^{m}+k\left\|\left(x^{(k)}\right)^{-}\right\|^{m} \leq 0 .
$$


Clearly, $\left\|x^{(k)}\right\|>0$. Let $y^{(k)}=\frac{x^{(k)}}{\left\|x^{(k)}\right\|}$. Then we have

$$
\mathcal{A}\left(y^{(k)}\right)^{m}+k\left\|\left(y^{(k)}\right)^{-}\right\|^{m} \leq 0 \text { for all positive integer } k,
$$

and hence,

$$
\frac{\mathcal{A}\left(y^{(k)}\right)^{m}}{k}+\left\|\left(y^{(k)}\right)^{-}\right\|^{m} \leq 0 \text { for all positive integer } k .
$$

Since $\left\|y^{(k)}\right\|=1$ for all positive integer $k$, we may assume that the sequence $\left\{y^{(k)}\right\}$ strongly converges to some vector $y \in \mathbb{R}^{n} \backslash\{0\}$ with $\|y\|=1$ (extracting a subsequence if necessary). Let $k \rightarrow \infty$ in (3.3). Then we have $\left\|y^{-}\right\|=0$, and so $y \in \mathbb{R}_{+}^{n} \backslash\{0\}$. It follows from the strict copositivity of $\mathcal{A}$ that

$$
\mathcal{A} y^{m}>0 \text {. }
$$

Since $\lim _{k \rightarrow \infty} \mathcal{A}\left(y^{(k)}\right)^{m}=\mathcal{A} y^{m}$, there exists a positive integer $N$ such that

$$
\mathcal{A}\left(y^{(k)}\right)^{m}>0 \text { for all } k>N .
$$

This yields a contradiction of (3.2). Thus there is $\gamma \geq 0$ such that the inequality (3.1) holds.

Conversely, take $x \in \mathbb{R}_{+}^{n} \backslash\{0\}$. Obviously, $\left\|x^{-}\right\|=0$. Therefore, it follows from the inequality (3.1) that

$$
\mathcal{A} x^{m}>0 \text { for all } x \in \mathbb{R}_{+}^{n} \backslash\{0\} .
$$

Therefore, $\mathcal{A}$ is strictly copositive, as required.

When $\mathcal{A}$ is a symmetric tensor of even order, $x^{-}$may be replaced by $x^{+}$ in Theorem 3.2.

Theorem 3.3. Let $\mathcal{A}$ be a symmetric tensor of order $m$ and dimension $n$. If $m$ is an even number, then $\mathcal{A}$ is strictly copositive if and only if there is a real number $\gamma \geq 0$ such that

$$
\mathcal{A} x^{m}+\gamma\left\|x^{+}\right\|^{m}>0, \text { for all } x \in \mathbb{R}^{n} \backslash\{0\} .
$$

Proof. Let $\mathcal{A}$ be strictly copositive. Since $m$ is an even number,

$$
\mathcal{A}(-x)^{m}=\mathcal{A} x^{m} \text {. }
$$

Suppose that for any positive integer $k$, there exists $x^{(k)} \in \mathbb{R}^{n} \backslash\{0\}$ such that

$$
\mathcal{A}\left(x^{(k)}\right)^{m}+k\left\|\left(x^{(k)}\right)^{+}\right\|^{m} \leq 0 .
$$

Take $y^{(k)}=\frac{x^{(k)}}{\left\|x^{(k)}\right\|}$. Then we have

$$
\frac{\mathcal{A}\left(y^{(k)}\right)^{m}}{k}+\left\|\left(y^{(k)}\right)^{+}\right\|^{m} \leq 0 \text { for all positive integer } k .
$$

Without loss of generality, we may assume that the sequence $\left\{y^{(k)}\right\}$ strongly converges to some vector $y \in \mathbb{R}^{n} \backslash\{0\}$ with $\|y\|=1$. Let $k \rightarrow \infty$ in (3.5). 
Then we have $\left\|y^{+}\right\|=0$, and so, $y \in \mathbb{R}_{-}^{n} \backslash\{0\}$ and $-y \in \mathbb{R}_{+}^{n} \backslash\{0\}$. It follows from the strict copositivity of $\mathcal{A}$ that

$$
\mathcal{A} y^{m}=\mathcal{A}(-y)^{m}>0
$$

Since $\lim _{k \rightarrow \infty} \mathcal{A}\left(y^{(k)}\right)^{m}=\mathcal{A} y^{m}$, there exists a positive integer $N$ such that

$$
\mathcal{A}\left(y^{(k)}\right)^{m}>0 \text { for all } k>N .
$$

This yields a contradiction of (3.5). Thus there is $\gamma \geq 0$ such that the inequality (3.4) holds.

Conversely, take $x \in \mathbb{R}_{+}^{n} \backslash\{0\}$. Obviously, $-x \in \mathbb{R}_{-}^{n} \backslash\{0\}$ and $\left\|(-x)^{+}\right\|=0$. Therefore, it follows from the inequality (3.4) that

$$
\mathcal{A} x^{m}=\mathcal{A}(-x)^{m}+\gamma\left\|(-x)^{+}\right\|^{m}>0 \text { for all } x \in \mathbb{R}_{+}^{n} \backslash\{0\} .
$$

Therefore, $\mathcal{A}$ is strictly copositive, as required.

Using similar proof of Proposition 3.1, we also easily prove the following conclusions.

Theorem 3.4. Let $\mathcal{A}$ be a symmetric tensor of order $m$ and dimension $n$. Then $\mathcal{A}$ is strictly copositive if and only if there is a real number $\gamma \geq 0$ such that

$$
\mathcal{A} x^{m}+\gamma\left\|x^{-}\right\|^{m}>0, \text { for all } x \in \mathbb{R}^{n} \text { with }\|x\|=1 .
$$

Theorem 3.5. Let $\mathcal{A}$ be a symmetric tensor of order $m$ and dimension $n$. If $m$ is an even number, then $\mathcal{A}$ is strictly copositive if and only if there is a real number $\gamma \geq 0$ such that

$$
\mathcal{A} x^{m}+\gamma\left\|x^{+}\right\|^{m}>0, \text { for all } x \in \mathbb{R}^{n} \text { with }\|x\|=1 .
$$

\section{Principal Sub-tensors of Copositive Tensors}

In homogeneous polynomial $\mathcal{A} x^{m}$ defined by (1.2), if we let some (but not all) $x_{i}$ be zero, then we have a less variable homogeneous polynomial, which defines a lower dimensional tensor. We call such a lower dimensional tensor a principal sub-tensor of $\mathcal{A}$, i.e., an $m$-order $r$-dimensional principal sub-tensor $\mathcal{B}$ of an $m$-order $n$-dimensional tensor $\mathcal{A}$ consists of $r^{m}$ elements in $\mathcal{A}=\left(a_{i_{1} \cdots i_{m}}\right)$ : for any set $\mathcal{N}$ that composed of $r$ elements in $\{1,2, \cdots, n\}$,

$$
\mathcal{B}=\left(a_{i_{1} \cdots i_{m}}\right), \text { for all } i_{1}, i_{2}, \cdots, i_{m} \in \mathcal{N} \text {. }
$$

The concept were first introduced and used by Qi [18] for the higher order symmetric tensor. Now we will continue to study the properties of the (strictly) copositive tensors by means of the principal sub-tensor of higher order symmetric tensor.

Theorem 4.1. Let $\mathcal{A}$ be a symmetric tensor of order $m$ and dimension $n$. Then $\mathcal{A}$ is copositive if and only if every principal sub-tensor of $\mathcal{A}$ has no negative $H^{++}$-eigenvalue, i.e., every principal sub-tensor of $\mathcal{A}$ has no eigenvector $v>0$ with associated $H$-eigenvalue $\lambda<0$. 
Proof. Let $\mathcal{A} x^{m} \geq 0$ for all $x \geq 0$. Suppose there exists an $m$-order $l$ dimensional principal sub-tensor $\mathcal{B}$ of $\mathcal{A}$ with an $H^{++}$-eigenvalue $\lambda<0$ $(1 \leq l \leq n)$, i.e. there is a positive vector $v \in \mathbb{R}_{++}^{l}$ such that

$$
\mathcal{B} v^{m-1}=\lambda v^{[m-1]} \text {. }
$$

Without loss of generality, we may write $v=\left(v_{1}, v_{2}, \cdots, v_{l}\right)^{T}\left(v_{i}>0\right.$ for $i=1, \cdots, l), x_{0}=\left(v_{1}, v_{2}, \cdots, v_{l}, 0, \cdots, 0\right)^{T}$. Then by the definition of principal sub-tensor, we have

$$
\mathcal{A} x_{0}^{m}=\mathcal{B} v^{m}=\lambda \sum_{i=1}^{l} v_{i}^{m}<0 .
$$

This contradicts the hypothesis that $\mathcal{A}$ is copositive. So, no principal subtensor of $\mathcal{A}$ can have a negative $H^{++}$-eigenvalue.

Conversely, let the principal sub-tensor of $\mathcal{A}$ have the property said in the theorem. Suppose that $\mathcal{A}$ is not copositive, then at least there exists a $y_{0} \geq 0$ such that $\mathcal{A} y_{0}^{m}<0$. Clearly, $y_{0} \neq 0$. Since the function $f(x)=A x^{m}$ is continuous and the set $K=\left\{x \in \mathbb{R}_{+}^{n} ; \sum_{i=1}^{n} x_{i}^{m}=1\right\}$ is a compact subset of $\mathbb{R}^{n}$, there is $y \in K$ such that

$$
f(y)=\mathcal{A} y^{m}=\min _{x \in K} \mathcal{A} x^{m} .
$$

Obviously, $f(y)=\mathcal{A} y^{m} \leq \mathcal{A} y_{0}^{m}<0$, and so some, but not all, components of $y$ may be 0 . Without loss of generality, we may assume that $y_{i}>0$ for $i=1,2, \cdots, l(1 \leq l \leq n)$ and $y=\left(y_{1}, y_{2}, \cdots, y_{l}, 0, \cdots, 0\right)^{T}$, and write $w=\left(y_{1}, y_{2}, \cdots, y_{l}\right)^{T}>0$. Let $\mathcal{B}$ be a principal sub-tensor that obtained from $\mathcal{A}$ by the polynomial $\mathcal{A} x^{m}$ for $x=\left(x_{1}, x_{2}, \cdots, x_{l}, 0, \cdots, 0\right)^{T}$. Then

$$
w \in \mathbb{R}_{++}^{l}, \sum_{i=1}^{l} y_{i}^{m}=1 \text { and } f(y)=\mathcal{A} y^{m}=\mathcal{B} w^{m}<0 .
$$

Let $x=\left(z_{1}, z_{2}, \cdots, z_{l}, 0, \cdots, 0\right)^{T} \in \mathbb{R}^{n}$ for all $z=\left(z_{1}, z_{2}, \cdots, z_{l}\right)^{T} \in \mathbb{R}^{l}$ with $\sum_{i=1}^{l} z_{i}^{m}=1$. Clearly, $x \in K$, and hence, by (4.1) and (4.2), we have

$$
f(x)=\mathcal{A} x^{m}=\mathcal{B} z^{m} \geq f(y)=\mathcal{A} y^{m}=\mathcal{B} w^{m} .
$$

Since $w \in \mathbb{R}_{++}^{l}, w$ is a local minimizer of the following optimization problem

$$
\begin{aligned}
& \min _{z \in \mathbb{R}^{l}} \mathcal{B} z^{m} \\
& \text { s.t. } \sum_{i=1}^{l} z_{i}^{m}=1 .
\end{aligned}
$$

So, the standard KKT conditions implies that there exists $\mu \in \mathbb{R}$ such that

$$
\nabla\left(\mathcal{B} z^{m}\right)-\left.\mu \nabla\left(\sum_{i=1}^{l} z_{i}^{m}-1\right)\right|_{z=w}=m \mathcal{B} w^{m-1}-m \mu w^{[m-1]}=0,
$$


and hence

$$
\mathcal{B} w^{m-1}=\mu w^{[m-1]} \text { and } \mathcal{B} w^{m}=\mu \sum_{i=1}^{l} y_{i}^{m}=\mu<0 .
$$

This implies that the negative real number $\mu$ would be an $H^{++}$-eigenvalue of a principal sub-tensor $\mathcal{B}$ of $\mathcal{A}$. By hypothesis, it's out of the question. So, $\mathcal{A} x^{m} \geq 0$ for all $x \geq 0$, as required.

Using the same proof as that of Theorem 4.1 with appropriate changes in the inequalities. We can obtain the following conclusions about the strictly copositive tensor.

Theorem 4.2. Let $\mathcal{A}$ be a symmetric tensor of order $m$ and dimension $n$. Then $\mathcal{A}$ is strictly copositive if and only if every principal sub-tensor of $\mathcal{A}$ has no non-positive $\mathrm{H}^{++}$-eigenvalue, i.e., every principal sub-tensor of $\mathcal{A}$ has no eigenvector $v>0$ with associated $H$-eigenvalue $\lambda \leq 0$.

Theorem 4.3. Let $\mathcal{A}$ be a symmetric tensor of order $m$ and dimension $n$. Then $\mathcal{A}$ is strictly copositive if and only if every principal sub-tensor of $\mathcal{A}$ has no non-positive $Z^{++}$-eigenvalue, i.e., every principal sub-tensor of $\mathcal{A}$ has no eigenvector $v>0$ with associated $Z$-eigenvalue $\lambda \leq 0$.

Proof. Let $\mathcal{A} x^{m}>0$ for all $x \geq 0$ with $x \neq 0$. Suppose there exists an $m$-order $k$-dimensional principal sub-tensor $\mathcal{B}$ of $\mathcal{A}$ with an $Z^{++}$-eigenvalue $\lambda \leq 0(1 \leq l \leq n)$, i.e. there is a positive vector $v \in \mathbb{R}_{++}^{l}$ such that

$$
\left\{\begin{array}{l}
\mathcal{B} v^{m-1}=\lambda v \\
v^{T} v=1
\end{array}\right.
$$

We may write $v=\left(v_{1}, v_{2}, \cdots, v_{l}\right)^{T}\left(v_{i}>0\right.$ for $\left.i=1, \cdots, l\right)$. Let $x_{0}=$ $\left(v_{1}, v_{2}, \cdots, v_{l}, 0, \cdots, 0\right)^{T}$. Then we have

$$
\mathcal{A} x_{0}^{m}=\mathcal{B} v^{m}=\lambda \sum_{i=1}^{l} v_{i}^{2}=\lambda \leq 0 .
$$

This contradicts the hypothesis. So, no principal sub-tensor of $\mathcal{A}$ can have a negative or zero $Z^{++}$-eigenvalue.

Conversely, let each principal sub-tensor of $\mathcal{A}$ have no non-positive $Z^{++}$eigenvalue. Suppose that $\mathcal{A}$ is not strictly copositive, then at least there exists a $y_{0} \geq 0$ with $y_{0} \neq 0$ such that $\mathcal{A} y_{0}^{m} \leq 0$. Since the function $f(x)=$ $A x^{m}$ is continuous and the set $S=\left\{x \in \mathbb{R}_{+}^{n} ; \sum_{i=1}^{n} x_{i}^{2}=1\right\}$ is a compact subset of $\mathbb{R}^{n}$, there is $y \in S$ such that

$$
f(y)=\mathcal{A} y^{m}=\min _{x \in S} \mathcal{A} x^{m} .
$$

Obviously, we must obtain that $f(y)=A y^{m} \leq \mathcal{A} y_{0}^{m} \leq 0$. Since $y_{0} \geq 0$ with $y_{0} \neq 0$, we may assume that $y=\left(y_{1}, y_{2}, \cdots, y_{l}, 0, \cdots, 0\right)^{T}\left(y_{i}>0\right.$ for $i=1, \cdots, l, 1 \leq l \leq n)$. Let $w=\left(y_{1}, y_{2}, \cdots, y_{l}\right)^{T}$ and let $\mathcal{B}$ be a 
principal sub-tensor that obtained from $\mathcal{A}$ by the polynomial $\mathcal{A} x^{m}$ for $x=$ $\left(x_{1}, x_{2}, \cdots, x_{l}, 0, \cdots, 0\right)^{T}$. Then

$$
w \in \mathbb{R}_{++}^{l}, \sum_{i=1}^{l} y_{i}^{2}=1 \text { and } f(y)=\mathcal{A} y^{m}=\mathcal{B} w^{m} \leq 0 .
$$

Let $x=\left(z_{1}, z_{2}, \cdots, z_{l}, 0, \cdots, 0\right)^{T} \in \mathbb{R}^{n}$ for all $z=\left(z_{1}, z_{2}, \cdots, z_{l}\right)^{T} \in \mathbb{R}^{l}$ with $\sum_{i=1}^{l} z_{i}^{2}=1$. Clearly, $x \in S$, and hence, by (4.3) and (4.4), we have

$$
f(x)=\mathcal{A} x^{m}=\mathcal{B} z^{m} \geq f(y)=\mathcal{A} y^{m}=\mathcal{B} w^{m} .
$$

Since $w \in \mathbb{R}_{++}^{l}, w$ is a local minimizer of the following optimization problem

$$
\begin{aligned}
& \min _{z \in \mathbb{R}^{l}} \mathcal{B} z^{m} \\
& \text { s.t. } \sum_{i=1}^{l} z_{i}^{2}=1 .
\end{aligned}
$$

So, the standard KKT conditions implies that there exists $\mu \in \mathbb{R}$ such that

$$
\begin{array}{r}
\left.\nabla\left(\mathcal{B} z^{m}-\mu\left(\sum_{i=1}^{l} z_{i}^{2}-1\right)\right)\right|_{z=w}=m \mathcal{B} w^{m-1}-2 \mu w=0, \text { and hence } \\
\mathcal{B} w^{m-1}=\frac{2 \mu}{m} w \text { and } \mathcal{B} w^{m}=\frac{2 \mu}{m} \sum_{i=1}^{l} y_{i}^{2}=\frac{2 \mu}{m} \leq 0 .
\end{array}
$$

This implies that the non-positive real number $\frac{2 \mu}{m}$ would be an $Z^{++}$-eigenvalue of a principal sub-tensor $\mathcal{B}$ of $\mathcal{A}$. By hypothesis, it's quite impossible. So, $\mathcal{A} x^{m}>0$ for all $x \geq 0$ with $x \neq 0$, as required.

Using the same proof as that of Theorem 4.3 with appropriate changes in the inequalities. We can obtain the following conclusions about the copositive tensor.

Theorem 4.4. Let $\mathcal{A}$ be a symmetric tensor of order $m$ and dimension $n$. Then $\mathcal{A}$ is copositive if and only if every principal sub-tensor of $\mathcal{A}$ has no negative $Z^{++}$-eigenvalue, i.e., every principal sub-tensor of $\mathcal{A}$ has no eigenvector $v>0$ with associated $Z$-eigenvalue $\lambda<0$.

Clearly, we have the following conclusions as a corollary of the above theorems.

Corollary 4.5. Let $\mathcal{A}$ be a symmetric tensor of order $m$ and dimension $n$. Then

(i) $\mathcal{A}$ is copositive if and only if for every principal sub-tensor $\mathcal{B}$ of $\mathcal{A}$, the fact that $\lambda$ is $\mathrm{H}^{++}$(or $Z^{++}$)-eigenvalue of $\mathcal{B}$ means that $\lambda \geq 0$;

(ii) $\mathcal{A}$ is strictly copositive if and only if for every principal sub-tensor $\mathcal{B}$ of $\mathcal{A}$, the fact that $\lambda$ is $H^{++}$(or $Z^{++}$)-eigenvalue of $\mathcal{B}$ means that $\lambda>0$. 
Corollary 4.6. Let $\mathcal{A}$ be a symmetric tensor of order $m$ and dimension $n$.

(i) If $\mathcal{A}$ is copositive, then $a_{i i \cdots i} \geq 0$ for all $i=1,2, \cdots, n$.

(ii) If $\mathcal{A}$ is strictly copositive, then $a_{i i \cdots i}>0$ for all $i=1,2, \cdots, n$.

(iii) Let $a_{i i \cdots i}=0$ for $i \in\{1,2, \cdots, n\}$. If $\mathcal{A}$ is copositive, then $a_{i i \cdots i j} \geq 0$ for all $j=1,2, \cdots, n$.

Proof. (i) For each fixed $i, \mathcal{B}_{i}=\left(a_{i i \cdots i}\right)$ is an $m$-order 1-dimensional principal sub-tensor $\mathcal{B}$ of $\mathcal{A}$. Clearly, $a_{i i \cdots i}$ is the unique eigenvalue with eigenvector (1). It follows from Theorem 4.1 (or 4.4) that $\mathcal{B}_{i}$ has no a negative $H^{++}$( or $Z^{++}$)-eigenvalue. Thus $a_{i i \cdots i} \geq 0$ for each $i \in\{1,2, \cdots, n\}$.

Similarly, from Theorem 4.2 (or 4.3), (ii) is easily obtained.

(iii) Let $\mathcal{B}_{i j}$ be a $m$-order 2 -dimensional principal sub-tensor of $\mathcal{A}$. Then by the definition of the principal sub-tensor, we have

$$
\mathcal{B}_{i j}=\left(a_{i_{1} i_{2} \cdots i_{k}}\right), \quad i_{1}, i_{2}, \cdots, i_{k}=i, j .
$$

Let $x=(t, 1)^{T}$ and $y=(t, 1,0,0, \cdots, 0)^{T}$ for $t>0$. Then

$$
\begin{aligned}
0 \leq \mathcal{A} y^{m}=\mathcal{B}_{i j} x^{m} & =t^{m} a_{i i \cdots i}+m t^{m-1} a_{i i \cdots i j}+\cdots+a_{j j \cdots j j} \\
& =m t^{m-1} a_{i i \cdots i j}+\left(\begin{array}{r}
2 \\
m
\end{array}\right) t^{m-2} a_{i i \cdots i j j}+\cdots+a_{j j \cdots j j} .
\end{aligned}
$$

So we have

$$
m t^{m-1} a_{i i \cdots i j} \geq-\left(\begin{array}{c}
2 \\
m
\end{array}\right) t^{m-2} a_{i i \cdots i j j}-\cdots-a_{j j \cdots j j},
$$

and hence

$$
a_{i i \cdots i j} \geq-\left(\begin{array}{c}
2 \\
m
\end{array}\right) \frac{a_{i i \cdots i j j}}{m t}-\cdots-\frac{a_{j j \cdots j j}}{m t^{m-1}} .
$$

Let $t \rightarrow \infty$. We have $a_{i i \cdots i j} \geq 0$.

It follows from the above results that the copositivity of tensors implies that all diagonal entry can not be negative, and the strict copositivity implies that all diagonal entry must be positive. So this reveals that in testing for (strict) copositivity of tensors, one can restrict attention to tensors with (positive) non-negative diagonal entries. We can further restrict attention to tensors whose diagonal entries are all equal to 1 ; we say that such a tensor has unit diagonal.

Example 1. Let $\mathcal{A}$ be a symmetric tensor of order 3 and dimension 2. If $a_{111}=a_{222}=0$, then it follows from Corollary 4.6 (iii) that $a_{i i j} \geq 0$ for $i, j=1,2$ whenever $\mathcal{A}$ is copositive. So $\mathcal{A}$ is copositive if and only if $\mathcal{A}$ is non-negative.

If $\mathcal{A}$ is copositive and $a_{111}=a_{222}=1$, then $a_{111}+a_{222}+3 a_{112}+3 a_{221} \geq 0$, and hence

$$
a_{112}+a_{221} \geq-\frac{2}{3}
$$


Example 2. Let $\mathcal{A}$ be a copositive and symmetric tensor of order 3 and dimension 3. If $a_{111}=a_{222}=a_{333}=0$, then

$$
a_{112} \geq 0, a_{113} \geq 0, a_{221} \geq 0, a_{331} \geq 0, a_{332} \geq 0, a_{223} \geq 0
$$

and

$$
2 a_{123}+a_{112}+a_{113}+a_{221}+a_{223}+a_{331}+a_{332} \geq 0 .
$$

If $a_{111}=a_{222}=a_{333}=1$, then consider an 3-order 2-dimensional principal sub-tensor of $\mathcal{A}$, we have

$$
a_{112}+a_{221} \geq-\frac{2}{3}, a_{113}+a_{331} \geq-\frac{2}{3}, a_{332}+a_{223} \geq-\frac{2}{3},
$$

and

$$
2 a_{123}+a_{112}+a_{113}+a_{221}+a_{223}+a_{331}+a_{332} \geq-1 .
$$

\section{ACKNOWLEDGMENTS}

The authors would like to express their sincere thanks to Dr. Lek-Heng Lim, the Handling Editor and the anonymous referees for their constructive comments and valuable suggestions.

\section{REFERENCES}

1. S. Burer, On the Copositive Representation of Binary and Continuous Nonconvex Quadratic Programs, Mathematical Programming, Series A, 120(2009) 479-495.

2. S. Burer and H. Dong, Representing Quadratically Constrained Quadratic Programs as Generalized Copositive Programs, Operations Research Letters, 40(2012) 203-206.

3. I. Bomze, Copositive optimization-recent developments and applications, European Journal of Operational Research, 216(2012) 509-520.

4. K. C. Chang, A nonlinear Krein Rutman theorem, J. Sys. Sci. Com. 22(4)(2009) 542554.

5. K.C. Chang, K. Pearson, and T. Zhang, Perron-Frobenius theorem for nonnegative tensors, Commun. Math. Sci. 6(2008) 507-520.

6. K.C. Chang, K. Pearson, T. Zhang, On eigenvalue problems of real symmetric tensors, J. Math. Anal. Appl. 350(2009) 416-422.

7. S. Hu and L. Qi, Algebraic connectivity of an even uniform hypergraph, J. Comb Optim, 24(4)(2012), 564-579.

8. S. Hu, Z. Huang and L. Qi, Finding the Spectral radius of a nonnegative tensor, arXiv:1111.2138v1 [math.NA] 9 Nov 2011.

9. E. Haynsworth and A.J. Hoffman, Two remarks on copositive matrices, Linear Algebra Appl. 2(1969) 387-392.

10. C.R. Johnson and R. Reams, Spectral theory of copositive matrices, Linear Algebra Appl. 395(2005) 275-281.

11. W. Kaplan, A test for copositive matrices, Linear Algebra Appl., 313(2000) 203-206.

12. L.H. Lim, Singular values and eigenvalues of tensors: A variational approach, in: Proc. 1st IEEE International workshop on computational advances of multi-tensor adaptive processing, Dec. 13-15, 2005, pp. 129-132.

13. D. H. Martin, Copositlve matrices and definiteness of quadratic forms subject to homogeneous linear inequality constraints, Linear Algebra Appl., 35(1981) 227-258.

14. T.S. Motzkin, quadratic forms, National Bureau of Standards Report,1818 (1952) 11-12.

15. G. Ni, L. Qi, F. Wang and Y. Wang, The degree of the E-characteristic polynomial of an even order tensor, J. Math. Anal. Appl. 329(2007) 1218-1229. 
16. M. Ng, L. Qi and G. Zhou, Finding the largest eigenvalue of a non-negative tensor, SIAM J. Matrix Anal. Appl., 31 (2009) 1090-1099.

17. L. Ping and F. Y. Yu, Criteria for Copositive Matrices of Order Four, Linear Algebra Appl. 194(1993) 109-124.

18. L. Qi, Eigenvalues of a real supersymmetric tensor, J. Symbolic Comput. 40(2005) 1302-1324.

19. L. Qi,Rank and eigenvalues of a supersymmetric tensor, the multivariate homogeneous polynomial and the algebraic hypersurface it defines, J. Symbolic Comput. 41(2006) 1309-1327.

20. L. Qi, Eigenvalues and invariants of tensors, J. Math. Anal. Appl. 325(2007) 13631377.

21. L. Qi, $H^{+}$-eigenvalues of Laplacian and signless Laplacian tensors, Preprint, Department of Applied Mathematics, The Hong Kong Polytechnic University, November, 2012. arXiv:1303.2186 [math.SP] 9 Mar 2013

22. L. Qi, Symmetric Nonnegative Tensors and Copositive Tensors, Linear Algebra Appl., 439(2013) 228-238.

23. Y. Song and L. Qi, Positive eigenvalue-eigenvector of nonlinear positive mappings, to appear in: Frontiers of Mathematics in China.

24. Y. Song and L. Qi, The existence and uniqueness of eigenvalues for monotone homogeneous mapping pairs, Nonlinear Analysis, 75(13)(2012) 5283-5293.

25. H. Väliaho, Criteria for copositive matrices, Linear Algebra Appl. 81(1986) 19-34.

26. Y. Yang and Q. Yang, Further Results for Perron-Frobenius Theorem for Nonnegative Tensors, SIAM J. Matrix Analysis Applications, 31(5)(2010) 2517-2530.

27. Q. Yang and Y. Yang, Further Results for Perron-Frobenius Theorem for Nonnegative Tensors II, SIAM Journal on Matrix Analysis and Applications 32(4)(2011) 1236-1250.

28. T. Zhang, Existence of real eigenvalues of real tensors, Nonlinear Analysis, 74(8)(2011) 2862-2868.

29. L. Zhang and L. Qi, Linear convergence of an algorithm for computing the largest eigenvalue of a nonnegative tensor, Numerical Linear Algebra with Applications, 19(2012) 830-841.

30. L. Zhang, L. Qi and Y. Xu, Linear convergence of the LZI algorithm for weakly positive tensors, Journal of Computational Mathematics, 30(2012) 24-33. 